\title{
UKRAINISCHER PÄDAGOGISCHER DISKURS: LINGUISTISCHE HISTORISCHE AUSLEGUNG
}

\section{Kravets L. V.}

\section{EINFÜHRUNG}

Der pädagogische Diskurs zeichnet sich durch soziolinguistische Merkmale aus und gilt als eine der Arten des institutionellen Diskurses ${ }^{1}$. Zahlreiche Studien zu diesem Problem in der ukrainischen und fremden Linguistik belegen die Mehrdeutigkeit der Interpretation des Begriffs "Diskurs". T. van Dijk betont, dass nur komplexe Theorien und sogar ganze Wissenschaftszweige die Bedeutung dieses Begriffs offenbaren können ${ }^{2}$. Erstens ist es durch die Natur des untersuchten Objekts verursacht, dessen Verständnis ein tiefes, facettenreiches Wissen über das menschliche Bewusstsein beinhaltet, das im Allgemeinen die Relevanz des Diskursstudiums bestimmt. O. Selivanova fasst die Überprüfung verschiedener Konzepte zusammen und identifiziert vier Hauptdefinitionen des Diskurses, die nicht nur die Ansätze seiner Untersuchung, sondern auch die Entwicklung der Sprachtheorien widerspiegeln. Insbesondere wird der Diskurs interpretiert als (1) „ein im Leben versunkener Text“, (2) „eine geschlossene ganzheitliche Kommunikationssituation (Ereignis)“, (3) „ein Stil, die Grundlage der Sprachkommunikation“, (4) „ein Muster des Sprachverhaltens in einem bestimmten sozialen Bereich" ${ }^{\star 3}$.

In dem Aspekt der Sprachphilosophie kann der Diskurs als Objektivierung der Sprache in verschiedenen Bereichen der menschlichen Kommunikation angesehen werden. Diese Interpretation steht im Einklang mit dem soziolinguistischen Verständnis des Diskurses als Kommunikation von Menschen einer bestimmten sozialen Gruppe in einer typischen Sprachverhaltenssituation ${ }^{4}$. Diese typischen Situationen treten in regelmäßig wiederholten Kommunikationsbedingungen auf, die den Umfang, den Ort,

${ }^{1}$ Карасик В.И. Языковой круг: личность, концепты, дискурс. Москва : Гнозис, 2004. C. 234, 251.

${ }^{2}$ Dijk T.A., van. Discourse and Context: A Sociocognitive Approach. New York : Cambridge University Press, 2008. P. 4.

3 Селіванова О.О. Лінгвістична енциклопедія. Полтава : Довкілля-К, 2010. C. $120-121$.

${ }^{4}$ Карасик В.И. Языковой круг: личность, концепты, дискурс. Москва : Гнозис, 2004. C. 233. 
den Zweck der Kommunikation sowie den Status und die Rolle ihrer Teilnehmer bestimmen. Der Kommunikationsbereich und die dadurch verursachte Typologie der Kommunikationsteilnehmer bilden die Grundlage für die Isolation institutioneller und existenzieller Diskurstypen ${ }^{5}$. Der institutionelle Diskurs umfasst politische, administrative, religiöse, medizinische, geschäftliche, pädagogische und andere Kommunikationsbereiche, nach denen seine Arten bestimmt werden. Der existenzielle Diskurs ist im Alltag und in der informellen persönlichkeitsorientierten Kommunikation (künstlerische und philosophische Sphäre) fixiert.

Der institutionelle Diskurs ist historisch variabel, was nicht nur das Erscheinen oder Verschwinden bestimmter sozialer Institutionen verursacht hat, sondern auch deren Transformation. Zur gleichen Zeit werden die Leitfäden der sprachlichen zwischenmenschlichen Interaktion, die sich historisch in einem bestimmten Bereich menschlichen Tätigkeit entwickelt haben, zum Kern, der die kategorischen Merkmale des Diskurses bestimmt und seine Nachhaltigkeit gewährleistet. ${ }^{6}$. Das Vorhandensein eines unveränderlichen traditionellen Satzes von Normen und Kommunikationsregeln, die nach Tätigkeitsbereichen differenziert sind, ist die Grundlage für die Typisierung des Diskurses sowie für seine retrospektive Untersuchung.

Der pädagogische Diskurs als eine Art des institutionellen Diskurses zeichnet sich durch folgende Merkmale aus: typische Kommunikationsteilnehmer, Kommunikationsbedingungen (Voraussetzungen, Kommunikationsumgebung, Chronotopos), Zweck, Werte, Strategien, Genres, Präzedenzfalltexte, diskursive Formeln. Die Kommunikation im Bildungsbereich (hauptsächlich zwischen Lehrern und Schülern oder Studenten) ist eine spezielle Art der zwischenmenschlichen Geschäftskommunikation, die epistemische Normen, Weltanschauungen, mentale Modelle, moralische und ethische Prinzipien sowie Wertorientierungen der Ethnie widerspiegelt, die sich im Verlauf seiner historischen Entwicklung herauskristallisiert haben. Der ist die Grundzusammensetzung des Bildungsprozesses, das Haupthandbuch für den Wissenstransfer, die Bildung von Fähigkeiten und Fertigkeiten, die Ausbildung und Entwicklung des Schülers. Die moderne pädagogische Kommunikation ist gleichzeitig stabil und dynamisch; nachdenklich, strukturiert und frei, direkt; geprägt vom Einfluss der Tradition und offen für Veränderungen. Im schulpflichtigen Alter gebildete Kommunikationsmodelle werden im späteren Leben entscheidend. Daher ist

\footnotetext{
${ }^{5}$ Там само. С. 234.

${ }^{6}$ Там само. С. 245.
} 
ihr Studium im pädagogischen Aspekt wichtig für die Entwicklung von Kommunikationsfähigkeiten, die Entwicklung von kritischem Denken und Interaktionsfähigkeiten in dem Prozess der Vorbereitung der Schüler und Studenten $\mathrm{zu}$ den weiteren sozialen Aktivitäten. Das Studium der Sprachkommunikation im ukrainischen Bildungsbereich ist auch in sprachlicher und soziolinguistischer Hinsicht relevant und mit der intensiven Entwicklung dieser Bereiche verbunden; wobei das Interesse der Wissenschaftler an den Besonderheiten der lebhaften gesprochenen Sprache in verschiedenen Bereichen der menschlichen Interaktion hoch ist.

Das Ziel des Artikels ist es, die Entwicklung des ukrainischen pädagogischen Diskurses, als kontinuierlichen historischen Prozess zu charakterisieren und seine Determinanten zu klären.

Die Entwicklung von Bildung und Ausbildung in der Ukraine wird in kulturogenen Texten (Chroniken, poetische Werke, pädagogische Literatur, wissenschaftliche Arbeiten) reflektiert. Die Ergebnisse ihrer Analyse werden in zahlreichen Arbeiten zur Geschichte der Pädagogik vorgestellt. Gleichzeitig wurde die Entwicklung des ukrainischen pädagogischen Diskurses in der wissenschaftlichen Literatur noch nicht ausführlich behandelt. Die Besonderheiten der Quellenbasis und der Umfang der Publikation ermöglichen es, die sprachstatusorientierte Interaktion im Bildungsbereich während eines langen historischen Zeitraums nur allgemein zu charakterisieren.

\section{Der Beginn der Bildung des ukrainischen pädagogischen Diskurses}

Wir verbinden den Beginn der Bildung des ukrainischen pädagogischen Diskurses mit der Gründung der ersten Schulen in den ukrainischen Ländern nach der offiziellen Annahme des Christentums. Die konstitutiven Charakteristiken des Diskurses wurden schrittweise gebildet, aber ihr Auftreten wird in den frühen Stadien der Schulbildung aufgezeichnet. Die Kommunikation unter den neuen Bildungsbedingungen für die Ostslawen war gekennzeichnet durch die Anwesenheit typischer Teilnehmer (Lehrer Schüler), Chronotopos (relativ spezifische Zeit und Ort des Studiums), Zweck und Werte des Lernens, was im Allgemeinen auf die Entstehung von Pädagogik Diskurs hinweist. Der Fürst Wladimir der Große hat gleichzeitig die Kirchen gebaut und die Kircheschulen für den „Buchunterricht“ organisiert, deren Ziel es war, eine christliche Weltanschauung unter den Schülern zu bilden und christliche Werte zu etablieren. Wie die Erwähnungen bezeugen, erreichte die Bildung in Kiewer Rus während der Zeit Jaroslaws des Weisen den höchsten Entwicklungsstand. Es ist bekannt, dass es in der St. Sophia Kathedrale in Kiew eine Hofschule gab, in der Schreibe und Übersetzer ausgebildet und Bücher umgeschrieben wurden. Es gab auch Klosterschulen, in denen neu aufgenommene Mönche in streng asketischem Geist unterrichtet wurden, um Gehorsam und Geduld in ihnen 
$\mathrm{zu}$ fördern. Es gab auch alphabetisierungsschulen, Frauenschulen und Heimunterrichten, die durch gemäßigte Anforderungen und Disziplin geprägt wurden ${ }^{7}$. In den schriftlichen Referenzen der vormongolischen Zeit wurden die allgemeinen Regeln der sprachstatusorientierten Interaktion jener Zeit in Übereinstimmung mit den Anforderungen der christlichen Ethik formuliert („Die Predigt über Recht und Gnade“, „Hypatiuschronik“, „Das Leben von Wladimir" von Dimitrij Tuptalo). Insbesondere erhaltet „Belehrung des Wladimir Monomach“ die Ratschläge für jungen Menschen: „,vor den Alten zu schweigen, auf die Weisen zu hören, den Älteren zu gehorchen, mit Gleichen und Geringeren freundlich zu sein; ohne List zu sprechen, viel verstehen; sei nicht böse durch die Wörter, lästere nicht, lache nicht zu viel, sei schüchtern vor den Älteren..." ${ }^{8}$. Imperative Formen der verwendeten Verben implizieren die strikte Einhaltung von Ratschlägen. Gleichzeitig werden die Belehrungen nicht nach den Erfordernissen der religiösen und asketischen Erziehung, sondern mit einem Fokus auf die lebenswichtigen Bedürfnisse des Menschen formuliert. Viele dieser Richtlinien sind heute im ukrainischen pädagogischen Diskurs relevant, aber im Allgemeinen ändern sich die Regeln der sprachstatusorientierten Interaktion und die damit verbundenen diskursiven Formeln in der Bildung ständig entsprechend der Forderung der Gesellschaft nach einer bestimmten Art von Persönlichkeit, einer bestimmten Art von Kommunikation sozioökonomische und kulturelle Bedingungen, sowie religiöse Situationen.

Die Bildungstraditionen der Kiewer Rus wurden vom Fürstentum Galizien-Wolhynien geerbt, wie aus der Galizien-Wolhynien Chronik hervorgeht. Gleichzeitig mit der Entwicklung der Schulbildung in diesen Ländern bildet sich weiterhin ein pädagogischer Diskurs. Die Grundschulbildung wurde in Pfarrschulen bei den Kirchen erhalten, in denen die Rolle des Lehrers von Diakonen wahrgenommen wurde. Das pädagogische Ziel dieser Schulen war es, die religiösen Bücher lesen zu lernen, rechnen, und die Gläubigen zu erziehen. Wir erfassen das Vorhandensein eines Chronotopos, aber es ist nicht klar abgegrenzt: die Fristen des Unterrichts sowie die Dauer der Ausbildung, der Beginn und das Ende des Schuljahres sind ungewiss. Die Ausbildung in Klosterschulen war regulierter und bot eine bessere Qualität, weil dort nicht nur besser ausgebildete Mönchslehrer arbeiteten, sondern auch die Büchersammlungen vorhanden waren. Die Priesterkinder haben entweder die Heimschule oder die Klosterschule besucht. Die wohlhabenden Familien haben als der Erzieher und Lehrer einen ausgebildeten Diener gehabt, und ab Ende des 15. Jahrhunderts - einen angestellten Bachelor. Die Kommunikation (der Sprachstil, das Genre) hatte

\footnotetext{
7 Літопис руський / пер. Л.С. Махновця. Київ : Дніпро, 1989. С. 66.

${ }^{8}$ Там само. С. 456.
} 
allgemein einen beliebigen Charakter und erfolgte häufig in der Gesprächsform. Die Ausbildung der Bevölkerung in den ukrainischen Ländern war zu dieser Zeit zwar bedeutend, aber nicht weit verbreitet.

Ab Ende des 16. Jahrhunderts gab es ein akutes Problem mit der Art und Richtung der Bildung in dem ukrainischen Raum, was hat sich in der damaligen Poesie widerspiegelte. Nach Forschungen von V. Krekoten, ,am Ende des 16. Jahrhunderts und zu Beginn des 17. Jahrhunderts stand der mittelalterlichen traditionellen „klösterlichen“ Position im Ausbildungsbereich, eine „brüderschaftliche“ Position, die den Bedarf an moderner Bildung befriedigen sollte, gegenüber"9 . Mit dem Aufkommen des Latein-orientierte Kyiv-Mohyla-Kollegiums wurde eine neue Art von Ausbildung und Schulen in der Ukraine gegründet ${ }^{10}$. Die öffentliche Nachfrage nach besser ausgebildeten Menschen sowie der Wunsch verschiedener Richtungen des Christentums, den Kreis der Anhänger zu erweitern und ihren Einfluss in den ostslawischen Ländern zu stärken, entstanden neue Ausbildungsinstitutionen mit unterschiedlichen ideologischen Ausrichtungen. Insbesondere im 16. - 17. Jahrhundert in den ukrainischen Ländern gab es fünf Schultypen: die brüderlichen Schulen, die Pfarrschulen (die Klosterschulen und die Kirchenschulen), die Regimentsschulen, die Schulen der protestantischen Gemeinschaften (Calvinisten, Lutheraner, Arianer), die Schulen des katholischen Ordens (Jesuiten und Piaristen) und Schulen der anderen Nationen (Juden, Armenier $)^{11}$. Die pädagogische Tätigkeit und der entsprechende pädagogische Diskurs jeder Art hat eine gesonderte detaillierte Untersuchung verdient, aber wir konzentrieren uns auf die brüderlichen und jesuitischen Schulen, weil die in der ukrainischen Ausbildung die sichtbaren Spuren hinterlassen haben.

Die Bruderschaften und die Schulen entstanden (die berühmtesten sind die Schule der Bruderschaft in Lviv (1585 gegründet) und die Schule der Bruderschaft in Kyiv (1615 gegründet) während der Intensivierung des Kampfes zwischen Reformation und Gegenreformation, der Umstrukturierung der russisch-litauischen Gesellschaft und die Verschärfung sozioökonomischer und interethnischer Widersprüche. Die Entstehung der brüderschaftlichen Schulen ist hauptsächlich eine Reaktion auf die Eröffnung und die Aktivität der Jesuitenschulen in den ukrainischen Ländern. Die Ausbildungsaktivitäten der Bruderschaften zielten darauf ab, die Orthodoxie zu stärken und eine ethnospezifische Weltanschauung und

9 Крекотень В. Освітня реформа Петра Могили й утвердження бароко в українській поезії. Записки Наукового товариства імені Шевченка. Львів, 1992. Том CCXXIV. Праці Філологічної секції. С. 10.

${ }^{10}$ Там само.

${ }^{11}$ Микитась В. Давньоукраїнські студенти і професори. Київ : Абрис, 1994. С. 50. 
Kultur zu bilden. Dies bestimmten den Zweck und die Werte des pädagogischen Diskurses, die den religiösen und moralischen Traditionen früherer Epochen entsprachen und die westeuropäischen humanistischen Ideen widerspiegelte. In brüderschaftlichen Schulen herrschte eine strenge Disziplin, zu der sowohl die Ermutigung als auch eine körperliche Bestrafung und angemessene sprachorientierte Interaktion gehörten. Die Lehrer mussten hochmoralische Fachkräfte sein, und die Schüler mussten ehrlich, höflich und diszipliniert sein. Die Rechte und Pflichten von Didaktiker (Didascaloi - plural aus Altgriechisch Didascalos) und Schülern (Spudeos -aus Altgriechisch fleißig) sowie ihren Eltern sind in der Satzung „Schulordnung“ (1586) von der Schule der Bruderschaft in Lviv als die erste Satzung in der Ukraine festgelegt worden und diente als Grundlage für ähnliche Satzungen in anderen brüderschaftlichen Schulen. Insbesondere verpflichtete er die Lehrer Bildungsgespräche mit den Schülern zu führen, „um ihnen die Angst vor Gott zu nehmen und die richtigen jugendlichen Gepflogenheiten beizubringen: wie sollten sie sich in der Kirche vor Gott, zu Hause vor Verwandten und anderswo verhalten, sich schämen, Gott und seinen Heiligen die Ehre und die Angst, den Verwandten und Lehrern den Gehorsam und für alle die Demut und Respekt zeigen, um für sich selbst die Reinheit zu behalten “"12. Gleichzeitig mit den brüderschaftlichen Schulen in den ukrainischen Ländern fungierten weiterhin Pfarrschulen in Kirchen und Klöstern, was im Allgemeinen zur Verbreitung der Ausbildung beitrug.

Der pädagogische Diskurs des Mittelalters entwickelte sich innerhalb des religiösen Diskurses, sowohl die Schulen überwiegend kirchlich waren als auch der Unterricht in ihnen darauf abzielte um die Prinzipien der christlichen Religion zu studieren, um sie in der Gesellschaft zu fördern und zu etablieren. Die Ausbildungstätigkeit wurde unter den Bedingungen der Gegenreformation besonders wichtig, als im Katholizismus viele Mönchsgemeinschaften entstanden um gegen den Protestantismus zu kämpfen. Einer der aktivsten in diesem Kampf war der Jesuitenorden, der den katholischen Glauben verbreiten sollten und die Autorität der Kirche zu stärken. Die Hauptaktivitäten des christlichen Mönchsordens der römischkatholischen Kirche war die Evangelisierung, die Ausbildung und die Forschung. Der Gründer des Jesuitenordens, Ignatius von Loyola, betrachtete die pädagogische Tätigkeit als eine der Kernaufgaben der Religionsgemeinschaft, und das Grundprinzip der Erziehung war der Gehorsam und die Einhaltung der Regeln des Ordens. Das Studium an Jesuitenkollegium hat geistes- und philologische Ausbildung mit religiöser und moralischer Erziehung verknüpft. Die Jesuiten legten großen Wert auf

\footnotetext{
12 Львівська братська школа. URL: https://uk.wikipedia.org/wiki/ Львівська_братська_школа.
} 
die Sprache, die sie nicht nur als Ausdrucksform, Dekoration oder Instrument verstanden, sondern viel tiefer als ihre Fähigkeit, die inneren Ressourcen des Menschen zu aktivieren. Laut R. Bart hat Ignatius von Loyola ein neues System spiritueller Übungen erstellt und eine neue nichtsprachliche und nicht-kommunikative Sprache - die Sprache der Fragen entwickelt. „Wenn in natürlichen Sprachen die Elementarstruktur einer Phrase, die in einem Subjekt und einem Prädikat artikuliert ist, zur durchsetzungsfähigen Ordnung gehört, dann werden in Ignatius Fragen und Antworten artikuliert“"13. Die Jesuiten, die Erben und Verbreiter der lateinischen Rhetorik und Poetik in der Ausbildung, legten großen Wert auf die Kunst der Überzeugung und des Dialogs. Wie R. Barth betont, hat die Kirche ihre Autorität auf das Wort gestützt, weil der Glaube eine Anhörung ist. $^{14}$. Die Rhetorik und die Poetik wurden an Jesuitenkollegs auf hohem Niveau unterrichtet und Fähigkeiten zur Erstellung von Reden, dramatischen Dialogen und Theaterstücken entwickelt. Am Jesuitenkollegium wurden die Rhetorik und die Poetik auf hohem Niveau unterrichtet und die Kunstfertigkeit zur Erstellung von Reden, dramatischen Dialogen und Theaterstücken entwickelt.

Die Kombination von humanitärer und philologischer Ausbildung mit religiöser und moralischer Erziehung ist auch charakteristisch für das KyivMohyla-Kollegium, das eine synkretistisch-orthodox-lateinische Ausrichtung hatte. Im 17. Jahrhundert auf dem ukrainischen Territorium gab es ein Ausbildungssystem, das von pro-westlichen Kirchenhierarchen (hauptsächlich Petro Mohyla) nach westeuropäischen Vorbildern aufgebaut wurde. „Die Orientierung an katholischen Wissenschaftsmodellen, die lateinische Sprache des Schulunterrichts unter Wahrung der traditionell östlichen kirchlichen Bindungen schufen einzigartige kulturelle Bedingungen, unter denen die Vererbung mit der mittelalterlichen Kultur erhalten blieb und die Wissenschaft selbst in doktrinären religiösen Angelegenheiten einen säkularen oder halbsäkularen Charakter erhielt"“15.

In den westlichen ukrainischen Ländern war die Ausbildungsaktivität eines anderen römisch-katholischen Mönchsordens - der Piaristen spürbar, deren Hauptaufgabe darin bestand die Kinder zu unterrichten und zu erziehen. Josef von Calasanz (auch José de Calasanz), der Gründer des Ordens, sah das Ziel der Ausbildung nicht nur in der intellektuellen, sondern auch in der moralischen Entwicklung der Schüler, der mit dem Respekt vor ihnen erreicht wird. Die Besonderheit der piaristischen Ausbildung war der völlige Verzicht auf körperliche Bestrafung, aber die Ermunterungen und die

\footnotetext{
${ }^{13}$ Барт Р. Сад, Фурье, Лойола. Москва : Праксис, 2007. С. 62.

${ }^{14}$ Там само. С. 87.

${ }^{15}$ Попович М.В. Нарис історії культури України. Київ : АртЕк, 1998. С. 722.
} 
Belohnungen waren weit verbreitet. Die sprachstatusorientierte Interaktion in den Schulen der Piaristen war durch eine respektvolle Haltung der Lehrer vor den Schülern gekennzeichnet ${ }^{16}$.

Die verschiedenen Ausbildungssysteme, die im religiösen Umfeld existierten, wurden durch die Konzentration auf das Verhaltensideal vereint, was gute Sprachkenntnisse in Latein, die Frömmigkeit, die Demut, die Askese und der Gehorsam bedeutete. Um dieses Ziel zu erreichen, wurden hauptsächlich strenge Disziplin, der Zwang und schwere körperliche Bestrafung (die Schläge, längeres Knien, der Hunger) unternommen, die als angenehm für Gott angesehen wurden, aber es wurden auch bestimmte Aufmunterungen angewendet.

Das Vorhandensein eines funktional gekennzeichneten Wortschatzes kennzeichnet den damaligen pädagogischen Diskurs. Die Ausbildungsinstitutionen wurden nach dem Typen als die Schule oder Kollegium, seltener Akademien benannt. Manchmal hatte dieselbe Ausbildungsinstitution unterschiedliche Namen, zum Beispiel Ostroher Schule - Ostroh-Griechisch-Slawisch-Lateinisches Kollegium, Ostroh-und Griechisch-Slawische Oberschule, Griechisches Kollegium, dreisprachiges Lyzeum, dreisprachiges Gymnasium, Akademie ${ }^{17}$.

Die Grundlage des Ausbildungsprozess, der nach westeuropäischem Typ geordneten war, bestand aus 8 Klassen, deren Namen festgelegt wurden: Fara-Klasse (oder Analogie-Klasse, der Vorbereitungsklasse, die Schüler mussten drei Sprachen lesen und schreiben beherrschen), Infima, Grammatik und Syntax (als drei Grundklassen), Poesie, Rhetorik (als zwei Mittelklassen), Philosophie, Theologie (als zwei Abiturklassen). Die Klassenaufgaben wurden als Exerzitien und die Hausaufgaben als Okkupatien genannt.

Die Teilnehmer des Bildungsprozesses sind klar definiert - die Lehrer oder Didaktiker sowie die Professoren und die Schüler, Studenten / Spudeos, Bursaks.

In den ukrainischen Hochschulen gab es eine Rangfolge der Schüler nach seinem Erfolg und erfüllte Aufgaben, was etwas lustig war. Diese Aufteilung der Schüler wurde von den westeuropäischen Schulen mit der Erhaltung von Namen entlehnt. Zum Beispiel, unter den Studenten waren "Kaiser“, „Senator", „Auditor", „Auditor der Auditoren“, „Zensor"; in den Hochschulen ernannte „Senioren“, „Direktoren“, „Inspektoren" sowie

${ }^{16}$ Шеретюк Р. «Благочестя і освіта»: просвітницькі ідеї Йосифа де Каласанса та їхній вплив на духовно-культурне життя Волині (XVII - перша пол. XIX ст.). URL: http://nbuv.gov.ua/UJRN/apvtvi_2016_27_50. 1994. C. 51 .

Микитась В. Давньоукраїнські студенти і професори. Київ : Абрис, 
„Peitsche“, die „Birkenbrei“ ausgaben ${ }^{18}$. Einige dieser Namen blieben bis zum Ende des 19. Jahrhunderts im pädagogischen Diskurs, wie die Werke von I. Franko, A. Svydnytsky, I. Nechuy-Levytsky und anderen belegen.

Die Ideen das Zeitalter der Aufklärung bildeten die Grundlage der modernen Pädagogik und beeinflussten die Weiterentwicklung des pädagogischen Diskurses. Die Säkularisierungsprozesse, die sich in europäischen Ländern nach der Französischen Revolution in großem Umfang entwickelten, der Entdeckungen in der Wissenschaft, der Evolution von Wirtschaftssystemen, die eine soziale Nachfrage nach einem neuen Persönlichkeitstyp bildeten, trugen zur intensiven Entwicklung der säkularen Ausbildung und zur Entstehung innovativer pädagogischer Konzepte bei, die von den Ideen des Humanismus durchdrungen sind. Die Werke von Johann Amos Comenius, John Locke, Jean-Jacques Rousseau und anderen haben die Grundlage des neuen Ausbildungssystems gebildet. Johann Amos Comenius sah der Gewährleistung zu einem erfolgreichen Staat in der allgemeinen Ausbildung von Kindern und Jugendlichen und schlug von Kindheit an vor, die Gerechtigkeit, Umsicht (Vernünftigkeit) und Beharrlichkeit in der Arbeit zu erziehen. John Locke zog die Erziehung mit sanften Vorschlägen und freundlichen Worten vor und glaubte, dass man auf diese Weise positive Gewohnheiten entwickeln kann. Jean-Jacques Rousseau schuf ein kindzentriertes pädagogisches Programm, dessen Hauptprinzipien Respekt und Liebe für das Kind waren. Der Wissenschaftler lehnte Autoritarismus, Bestrafungs- und Zwangsmethoden im Bildungsprozess ab. Die Ansichten von Jean-Jacques Rousseau diente als die Grundlage für die Theorie der freien Erziehung, die von Maria Montessori und Alexander Neill weiterentwickelt wurde.

Die Schulen des Semstwos, die im späten 19. Jahrhundert in den ukrainischen Ländern des russischen Reiches auftauchten, versuchten die humanistischen pädagogischen Ideen umzusetzen. Aber die Empfehlungen an die Lehrer die Schüler freundlich und höflich zu behandeln, während der Ausbildung eine hohe Motivation aufrechtzuerhalten und keine disziplinarischen Sanktionen zu verhängen, wurden in der Praxis jedoch nicht immer implementiert. Im Allgemeinen blieb die schwere körperliche Bestrafung in Ausbildungsinstitutionen verschiedener Typen fast bis zum Beginn des 20. Jahrhunderts bestehen. Die Studienbedingungen spiegelten sich im damaligen pädagogischen Diskurs wider, in dem das von Lehrern und Professoren zur Ansprache von Schülern und Schülern verwendete invektiver Wortschatz keine Seltenheit war. Kirchenslawisch funktionierte in Schulen in den subrussischen Ländern. Die Besonderheiten der sprachstatusorientierten Interaktion in Ausbildungsinstitutionen Mitte des

${ }^{18}$ Там само. С. 70 . 
19. Jahrhunderts zeigt insbesondere Anatoliy Svydnytsky im Roman „Lyuboratski“. Im Vorwort zur Auflage der Werke des Schriftstellers stellt Petro Khropko fest: „Svydnytsky entschleiert und verurteilt das antipädagogische Unterricht- und Ausbildungssystem. Die täglichen körperlichen Foltern von Studenten, der Despotismus von ,älteren“ Bursaks, Betrug und Insinuation, die „Etiketten“ an diejenigen aufkleben, die „Bauer“ waren, d.h. ihre Muttersprache gesprochen haben, - so war das System, das Jugendliche verkrüppelte und verdarb“"19.

In den ukrainischen Ländern, die Teil des österreichischen Reiches waren (und seit 1867 Österreichisch-Ungarische Monarchie), war die Ausbildung etwas anders - es gab Normale-, Haupt- und Trivialschulen. Die Sekundarschulausbildung wurde in Gymnasium und Realschulen durchgeführt. In den westukrainischen Territorium vorherrschte die Pfarrschulen, unter denen der ukrainische pädagogische Diskurs erhalten blieb, da dort der Unterricht auf Ukrainisch durchgeführt wurde. Die Entwicklung des ukrainischen pädagogischen Diskurses in diesem Bereich wurde durch die Aktivitäten der Bildungsorganisation „Galizien-Russischen Matytsa“" (gegründet in 1848), der Abteilung der russischen Literatur an der Lviv Universität (gegründet in 1849), die Organisation „Proswita“ (gegründet in 1868), die Russische Pädagogische Gesellschaft (gegründet in 1881; in 1912 auf Ukrainische Pädagogische Gesellschaft und in 1926 auf „Ridna Schkola“ umbenannt) und Ländliche Schulunion („Krajovij Schulunion“) gefördert.

Der Ausbildungsprozess wird von gesellschaftspolitischen und wirtschaftlichen Faktoren bestimmt, die ihn in eine bestimmte Richtung lenken. Wenn in der Antike die Ausbildung ein Vorrecht der wohlhabenden Bevölkerungsgruppen war, dann bestand mit dem Aufkommen neuer Arbeitsbeziehungen die Notwendigkeit einer obligatorischen Massenbildung und einer ständigen Verbesserung der Wissensqualität. Die meisten Schulen konnten zu dieser Zeit die gestiegenen Anforderungen nicht erfüllen. $\mathrm{Zu}$ Hause oder in einer kirchlichen Schule erworbenes Wissen machte die weitere Sekundar - und Hochschulbildung für Absolventen unzugänglich. Die öffentliche Massenschule, die $\mathrm{zu}$ dieser Zeit auf dem neuen KlassenUnterrichtssystem beruhte und reglementiert wurde, wurde aufgefordert, eine große Anzahl von Lese- und Schreibkräften auszubilden, die im Industriezeitalter benötigt wurden. Der sprachliche statusorientierte Umgang, der Ausbildungsprozess in der Massengrundschule des 19. Jahrhunderts ist nach wie vor von Grausamkeit, Zwang, moralischer Demütigung der Schüler und schwerer Bestrafung geprägt. Iwan Franko hat die Proletarisierung des

19 Хропко П.П. Анатолій Свидницький. Анатолій Свидницький. Роман, оповідання, нариси / за ред. П.П. Хропка. Київ : Наукова думка, 1985. С. 20. 
galizischen Dorfes und die Atmosphäre der damaligen Schulbildung in einer Reihe von Kindergeschichten meisterhaft reproduziert. Das beschriebene Ausbildungsumfeld hat geholfen um einen gehorsamen, sanftmütigen, demütigen und bescheidenen Arbeiter vorzubereiten.

\section{Ukrainischer pädagogischer Diskurs der Neuzeit}

Der Beginn des 20. Jahrhunderts brachte radikale Veränderungen in das Leben des ukrainischen Volkes, die sich auf den Ausbildungsbereich auswirkten. Unter der Zentralrada, der Ukrainischen Volksrepublik (Ukrajinska Narodna Respublika - UNR) und dem Hetmanat gab es eine intensive Entwicklung der ukrainischen Bildung und damit des ukrainischen pädagogischen Diskurses. Grundschulen wurden eingerichtet, ein Ausbildungssystem für Erwachsene organisiert und ukrainische Universitäten eröffnet. Die führende Rolle in diesem Prozess spielte die Schulbildungsgesellschaft, die die ukrainische Terminologie entwickelte, Lehrpläne erstellte und zur Entstehung ukrainischer Schulbücher beitrug. Während der UNR Zeiten arbeiteten sie an der Schaffung einer 12-jährigen Arbeitsschule.

Nach der Errichtung der Sowjetmacht in der Ukraine wurden die Besonderheiten des ukrainischen pädagogischen Diskurses von der sowjetischen Ideologie bestimmt. Allerdings war dieser Zeitraum nicht homogen. Die Veränderungen im Bildungsbereich hingen von der gesellschaftspolitischen Situation im Land ab, von den Hauptrichtungen der Innenpolitik der regierenden Kommunistischen Partei. Die Entwicklung der Pädagogik als Wissenschaft hat ebenfalls mehrfache Richtungen gehabt. All dies spiegelte sich im pädagogischen Diskurs wider. Olha Sukhomlynska bemerkt: „Politische Klischees und Leitfäden haben sich oft gegen den tatsächlichen pädagogischen Inhalt und die Orientierung durchgesetzt. In all den Jahren der sowjetischen Pädagogik existierte, mit Ausnahme seiner frühen Phase, besteht ein direkter, sogar pädagogisch indirekter Zusammenhang zwischen Entscheidungen, Resolutionen der KPdSU über Bildung und Pädagogik, die sofort reagierten, auf sie „nachklang“ und sie mit pädagogischem Inhalt füllten, technologisierten“"20.

Die neue Gesellschaftsordnung führte zu einer radikalen Umgestaltung von Ausbildung und Erziehung: die Schule wurde von der Kirche getrennt, private Ausbildungsinstitutionen wurden verboten, die obligatorische kostenlose öffentliche Ausbildung wurde eingeführt, die gemeinsame Bildung von Kindern beiderlei Geschlechts wurde erlaubt, die körperliche Bestrafung von Schülern wurde abgeschafft und so weiter. Der pädagogische

20 Сухомлинська О. Радянська педагогіка як ідеологія: спроба історичної реконструкції. URL: https://core.ac.uk/download/pdf/32308395.pdf. C. 5. 
Diskurs spiegelt weitreichende Veränderungen in Gesellschaft und Ausbildung wider. Die auffälligsten Änderungen treten in der Lexik auf. Die Wörter, die die Realitäten der vorangegangenen Epoche bezeichnen, vor allem die kirchlich-religiöse Sphäre und die gesellschaftspolitische, werden allmählich nicht mehr gebraucht. Der Inhalt des Schlüsselbegriffs Schule in einem neuen Kontext wird durch antonymische Definitionen geteilt: alte, bourgeois, frühere und neue, proletarische, moderne. Die Schule der vorangegangenen Epoche wurde als Instrument der Klassenherrschaft der Bourgeoisie charakterisiert und die Schule der Sowjetzeit als Instrument der kommunistischen Transformation der Gesellschaft genannt. Statt verschwendeten Gymnasien, Lyzeen, Kollegien entstehen neue Schultypen, deren Richtungen sich in den Namen widerspiegeln: Arbeitsschule, Fabrikschule (Schule der Fabriklehre), Schule der ländlichen Jugend usw. Um den Analphabetismus in der Bevölkerung in den 20er und 30er Jahren des 20. Jahrhunderts $\mathrm{zu}$ beseitigen, wurden zahlreiche Liknep (die Eliminierung des Analphabetismus) Punkte und Kreise eingerichtet.

Radikale Veränderungen im pädagogischen Diskurs wurden durch die Einführung komplexer Programme (1923) in die Schulpraxis verursacht, die auf den Grundsätzen des Bolschewismus gebaut. Sie stellten sich eine starre Politisierung und Ideologisierung des Ausbildungsprozesses vor und zielten darauf ab, das Hauptziel zu erreichen - die Bildung eines neuen Mannes, eines Erbauers einer „helleren Zukunft“, eines Trägers höherer sozialer Werte, einer neuen kommunistischen Moral.

In den 20er Jahren des 20. Jahrhunderts bekam der pädagogische Diskurs im Zusammenhang mit der Entwicklung der ukrainischen Schulausbildung klare ukrainische Merkmale. Vor allem erschien die kodifizierte ukrainische Rechtschreibung (1928 eingeführt, in 1933 abgeschafft), nationale Branchen orientierte Systeme wurden entwickelt, terminologische Wörterbücher und Lehrbücher wurden zusammengestellt und veröffentlicht, der Unterricht an ukrainischen Schulen wurde hauptsächlich auf Ukrainisch durchgeführt. All dies schuf die Voraussetzungen für die Entstehung und aktive Verwendung neuer diskursiver Formeln. Die nationale Entwicklung entsprach der Strategie der Partei, wonach alle nationalen Errungenschaften künftig in den Dienst des Sozialismus gestellt werden sollten. Der pädagogische Diskurs spiegelte revolutionäre Veränderungen in Gesellschaft und Ausbildung, Experimente, die zu dieser Zeit den Bildungsbereich abdeckten, sowie die Wiederbelebung der nationalen Kultur wider. In den frühen 1930er Jahren wurde die Politik der Ukrainisierung gestoppt, die Ideologisierung und Politisierung sowie die Ausbildung von Studenten im Stil der sowjetischen Einzigartigkeit in Bildungseinrichtungen intensiviert.

Das sowjetische Ausbildungssystem erfüllte eine Staatsaufbaumission und bildete den sowjetischen (überethnischen) Mensch aus, dessen Aufgabe war es, den Plan des Aufbaus des Sozialismus umzusetzen, militärische und 
arbeitsrechtliche Heldentaten bei der Industrialisierung des Landes durchzuführen und das Land von Invasoren zu schützen, die Städte und Dörfer nach dem Ruin wieder aufzubauen, den Weltraum zu erobern usw. Im Menschen der kommunistischen Gesellschaft bildeten sich soziale Aktivität, politisches Bewusstsein, Opferfähigkeit, hohes kulturelles Niveau, Fleiß, kreative Einstellung zur Arbeit. Die Werte der Ausbildung entsprachen der sowjetischen Ideologie. Die Schlüsselkonzepte des pädagogischen Diskurses waren Gerechtigkeit, Gleichheit, Solidarität, Selbstlosigkeit, Fleiß, Ordnung, Ehrlichkeit, Wahrhaftigkeit, Ehre, Kollektivismus und andere. Die Namen universeller menschlicher Werte wurden jedoch mit Definitionen verwendet, die laut Larysa Masenko „zu ihrer semantischen Transformation führten". Solche Namen moralischer und ethischer Kategorien als „Gewissen“, „Moral“, „Ehre“ usw., mit Idiolexemen kombiniert, verloren ihre universelle Bedeutung und erlangten ideologische Semantik der Klassenpartei: „kommunistische Moral“, „arbeitendes Gewissen“, „proletarische Ehre“, „kollektive Farmdemokratie“621.

Die Kultivierung hoher (utopischer) Ideale, definiert durch den Moralkodex des Erbauers des Kommunismus, erfolgte unter gleichzeitiger Abwertung des Lebens einer individuellen, moralischen und spirituellen Nivellierung der Persönlichkeit, die die etablierten Ausdrücke widerspiegelte: „Denken Sie zuerst an die Heimat, dann über dich selbst“, „,Wir brauchen kein Brot - gib uns Arbeit", ,,Von jedem nach Fähigkeiten, zu jedem nach Arbeit". Die Lebensbedürfnisse und Probleme eines Durchschnittsmenschen waren im Kontext des Aufbaus des Kommunismus gering, und der persönliche Raum (sowohl physisch als auch psychisch) war fast nicht vorhanden - die sowjetische Gesellschaft versuchte, selbst das intime Leben des Menschen zu kontrollieren. Die sowjetische Schule bildete die „Räder und Zahnräder eines einzigen großen Mechanismus", wobei häufig Sprachstempel wie ,,junge Leninisten“, ,, alle als eine“, ,wie alle sein “, ,, du bist nicht besser als andere “, ,, selbstlose Arbeit" usw. verwendet wurden. Der pädagogische Diskurs war gefüllt mit Sowjetismen, Ideologen: Oktoberisten, Pionieren, Komsomol-Mitgliedern, kommunistischem Wandel, Pionier Kader, Komsomol-Aktivisten, atheistischer Erziehung, sowjetischer Erziehung, internationaler Erziehung, Idealen des Kommunismus / Sozialismus usw. Die sprachstatusorientierte Interaktion im sowjetischen Ausbildungsbereich ist im Allgemeinen durch Autoritarismus, Direktivität und Zwang gekennzeichnet. Dies spiegelt sich insbesondere in den imperativen Slogans wider, die ein obligatorisches Attribut von

21 Масенко Л. Мова радянського тоталітаризму. Київ : ТОВ «Видавництво "Кліо"», 2017. С. 29. 
Ausbildungsinstitutionen waren: „Lernen, lernen und wieder lernen!“, „Beschütze das Buch - eine Quelle des Wissens!“, „Helfen Sie Senioren!“ usw. Die sowjetische Pädagogik laut Olha Sukhomlynska „verkörperte die Ausbildungspolitik der Kommunistischen Partei“. Eine wichtige und bestimmende Komponente der sowjetischen Pädagogik war die Bildung und Festigung eins angemessenen Lexikons, das die ideologische Realität reflektieren und interpretieren sollte, d.h. die „sowjetische Sprache“ wurde für die sowjetische Pädagogik geschaffen ${ }^{22}$.

In der zweiten Hälfte des 20. Jahrhunderts führten die gesellschaftspolitische Lage des Landes, die wissenschaftliche Erforschung der Geisteswissenschaften und die Aktivitäten von Lehrer-Innovatoren zur Diversifizierung des ukrainischen sowjetischen pädagogischen Diskurses. Die Tätigkeit von Wassyl Suchomlynskyj verdient besondere Aufmerksamkeit. Das vom Lehrer-Forscher entwickelte System basierte auf den Prinzipien des Humanismus. Im Zentrum des Systems steht die Persönlichkeit des Kindes, das als der größte Wert anerkannt wird. Die kreative Tätigkeit des vereinten pädagogischen Kollektivs im Umgang mit den Eltern richtet sich nach seiner vielfältigen Ausbildung und Erziehung. Der pädagogische Diskurs von Wassyl Suchomlynskyj im Allgemeinen blieb sowjetisch, wie diskursive Formeln als ,die Treue zu hohen kommunistischen Idealen“, ,glückliche Kindheit“, „Unnachgiebigkeit gegenüber der Versklavung und Unterdrückung des Menschen durch den Menschen “, ,, die Bereitschaft um die Kraft und das Leben im Namen hoher Ideale zu geben " usw. anzeigen. Gleichzeitig ist sein pädagogischer Diskurs klar individualisiert und enthält die Phrasen als Erziehung der Menschenwürde, die geistige Welt des Kindes, das geistige Leben des Kindes, die emotionale Sensibilität des Kindes, die Erziehung zu Güte und Menschlichkeit, das Geistige Reichtum des Menschen, weise menschliche Liebe usw. Die Frequenzkonzepte als Muttersprache, Heimatland, Kinder, Arbeit, Buch, Natur, Güte, moralisches Ideal manifestieren die Werte, die vom Lehrer aktualisiert werden. Die sprachliche Interaktion, die Wassyl Suchomlynskyj in seinen Werken reproduziert, basiert auf den Prinzipien des gegenseitigen Respekts zwischen Lehrer und Schüler. Der Forscher bevorzugt Erziehung mit Schönheit und harter Arbeit und versucht im Geiste der Zeit, „den unbeschwerten Genuss der Vorteile des Lebens zu verhindern" ${ }^{23}$.

22 Сухомлинська О. Радянська педагогіка як ідеологія: спроба історичної реконструкції. URL: https://core.ac.uk/download/pdf/32308395.pdf. C. 4.

${ }^{23}$ Сухомлинський В. Серце віддаю дітям. URL: https://mala.storinka.org/ серцевіддаю-дітям-василь-сухомлинський-читати-завантажити.html 
So war die Entwicklung des ukrainischen pädagogischen Diskurses in der Sowjetzeit komplex und ungleichmäßig, durch widersprüchliche Tendenzen gekennzeichnet, von Ideologie und Politik beeinflusst.

\section{Ukrainischer pädagogischer Diskurs der postsowjetischen Ära}

Der ukrainische pädagogische Diskurs der postsowjetischen Zeit war seit langem mit Merkmalen charakterisiert, erworben während des Totalitarismus. Die Veränderungen in der Ausbildung und im pädagogischen Diskurs wurden durch das längst überfällige Bedürfnis nach einer neuen Persönlichkeit in der ukrainischen Gesellschaft bestimmt, die auf der Grundlage nationaler und europäischer Werte gebildet wurde. Das vorrangige Prinzip des Humanismus in der modernen Welt, nach dem ein Mensch den höchsten Wert hat, seine Interessen und Bedürfnisse unter Berücksichtigung nationaler, rassistischer und religiöser Besonderheiten, ist es zu einem Schlüssel in der ukrainischen Ausbildung geworden. Die Orientierung an diesem Prinzip spiegelt sich im modernen ukrainischen pädagogischen Diskurs wider. Höflichkeit (Freundlichkeit, Gutwilligkeit), Takt, Korrektheit und Genauigkeit, die allmählich zur Norm der sprachlichen zwischenmenschlichen Interaktion in der Ausbildung werden, zeigen Respekt für die Würde jedes Menschen, erleichtern das gegenseitige Verständnis und Lernen. Die Kommunikation im Bildungssystem, die auf diesen Kriterien aufbaut, ist die Grundlage für die Bildung und Entwicklung gesunder Beziehungen in der gesamten Gesellschaft. Die Sätze als „Ich freue mich, Sie in diesem Raum begrüßen zu dürfen“, „Ich freue mich, Sie wiederzusehen “, ,Sie machen es gut “, ,, Gute Antwort “, „, Sie sind nahe an der Wahrheit", „Danke für die Antwort (Klarstellungen, Ergänzungen usw.) “, „,versuchen Sie es erneut, Sie werden definitiv Erfolg haben “, „Ich weiß, dass Sie erfolgreich damit umgehen können", "Sie machen es großartig", „Ich freue mich für Sie“, „Danke, dass Sie bei uns sind" ermutigen die Schüler und schaffen einen positiven emotionalen Hintergrund beim Lernen. In Äußerungen werden muss-Konstruktionen öfter durch Wunsch-Konstruktionen ersetzt (,,Bitte behalten Sie die Zeit im Auge und kommen Sie nicht zu spät“, „Bitte schalten Sie das Handy aus “ usw.), was dazu beiträgt, eine freundschaftliche Partnerschaft zwischen Lehrern und Schülern aufrechtzuerhalten. Die Fragen als „,Wie siehst du das?", ,Wie hast du es vorher gemacht? “, ,, Wie kannst du es sonst machen? “ aktivieren das Denken und die Kreativität, ermöglichen den Schülern ihre Stärken zu zeigen und eröffnen eine neue Perspektive für das Lernen. Sprachstatusorientierte Interaktion, Ausbildungsprozess in modernen ukrainischen Ausbildungsinstitutionen sind zunehmend durch gegenseitigen Respekt, Toleranz und Partnerschaften gekennzeichnet, die sich in jedem Sprachschritt des Diskurszyklus erkannt werden: Lehrerinitiative (Fragen, Förderung der Sprachinteraktion, die Bereitstellung die Möglichkeit zu 
antworten), Antworten der Schüler, Reaktionen der Lehrer auf die Antworten der Schüler.

Das humanistische Grundprinzip des menschlichen Selbstwertgefühls hat die Werte des modernen ukrainischen pädagogischen Diskurses bestimmt Ehre, Würde, Freiheit, Selbstversorgung, Selbstentwicklung, Zusammenarbeit, aktive bürgerliche Position, Patriotismus.

In der postsowjetischen Ära änderte sich die Typologie der Ausbildungsinstitutionen und es spiegelte ihre Namen wider: Schule (Allgemeinbildungsschule), Gymnasium, Lyzeum, College, Institut, Akademie, Universität usw.

In neue Ausbildungsbedingungen bleiben traditionell der Lehrer und der Schüler als die Teilnehmer des pädagogischen Diskurses, aber die Rolle beider Teilnehmer ändert sich erheblich. Wenn es nach dem vorherigen Ausbildungsparadigma die Pflicht des Lehrers war um das Wissen zu übertragen, dann nach dem neuen Paradigma ist es um die Schüler in die aktive Wissensproduktion einzubeziehen. Die berufliche Tätigkeit eines Lehrers sowie der gesamte Ausbildungsprozess sind persönlich ausgerichtet und zielen eher auf die Entwicklung des Denkens und der Kreativität der Schüler, die Bildung ihrer Fähigkeiten und Fertigkeiten zur Zusammenarbeit als auf wettbewerbsorientiertem Individualismus ab. Eine klare Unterscheidung zwischen bestimmten Arten von psychologischen und pädagogischen Aktivitäten hat zu einer Erweiterung der Liste der relevanten Namen geführt: Pädagoge, Lehrer, Erzieher, Lektor, Mentor, Berater, Tutor, Betreuer, Coach, Trainer, Moderator, Ausbilder, Anleiter, Ordinarius, Dozent, Professor. Die traditionelle Liste der Namen von Bewerber für die Ausbildung als Schüler, Schulkind, Lehrling, Student, Pennäler, Zuhörer, Kadett und Praktikant wurde durch die Wörter Bachelor, Meister, Gymnasiast und Auszubildende ergänzt, die aus dem passiven Lexikon zurückgekehrt waren.

Mit der Einführung digitaler Technologien im Ausbildungsprozess enthielt die Sprache von Lehrern, Schülern und Studenten zahlreiche Namen, um neue Realitäten zu kennzeichnen: interaktive Tafel, MultimediaKlasse, Tablet, E-Journal (elektronische Zeitschriften oder digitale Zeitschriften), elektronisches Tagebuch, elektronischer Zeitplan usw. Die Terminologie Phrasen als Ausbildungsportal, Ausbildungszentrum, Ausbildungsplattform erschienen. Die Trends der ukrainischen Ausbildung sind zu Personalisierung, Digitalisierung und Gamification geworden.

Mit dem Übergang zum Fernunterricht in Quarantäne Bedingungen haben sich im pädagogischen Diskurs extrem schnelle und weitreichende Veränderungen ergeben. Als der Bildungsprozess in den Online-Bereich verlagert wurde, hat sich das Chronotop des pädagogischen Diskurses, d.h. die Zeit und der Ort des Unterrichts, geändert. Die Wörter als virtuelle Plattform, Classroom (Klassenzimmer), Moodle, Zoom, Webinar, Training 
usw. wurden als Teil des täglichen Gebrauchs der Teilnehmer am Ausbildungsprozess eingebracht.

Die moderne ukrainische Ausbildung konzentriert sich auf der Persönlichkeit, persönlicher Wünsche und beinhaltet die aktive Teilnahme am Lernprozess der Schüler, die Zusammenarbeit zwischen Lehrern und Schülern, das Experimentieren, die Entwicklung kritischen Denkens und die Kreativität.

Eine sprachstatusorientierte Interaktion, die auf dem Prinzip des Humanismus basiert, gewährleistet die Erhaltung der psychischen Gesundheit der Kommunikationsteilnehmer und fördert die Bildung einer modernen ukrainischen Persönlichkeit. Die im Lernprozess erworbenen Modelle des Sprachverhaltens und der Kommunikationskompetenz sind in allen Bereichen des sozialen Lebens eins Erwachsenen entscheidend.

\section{SCHLUSSFOLGERUNGEN}

Der ukrainische pädagogische Diskurs hat sich während der Bildung und Entwicklung der ukrainischen Ausbildung von der Zeit der Kiewer Rus bis heute gebildet. Wir definieren den ukrainischen pädagogischen Diskurs hauptsächlich nach ethnospezifischen Charakteristiken, nämlich dem Territorium und das Volk, das dieses Territorium bewohnt hat, seiner Sprache und Kultur. Im Verlauf der historischen Entwicklung in der Gesellschaft wurde die Ausbildungssphäre identifiziert und die Regeln der sprachlichen zwischenmenschlichen Interaktion in diesem Bereich aufgestellt, die sich zwar weiterentwickelt haben, aber Hauptmerkmale des institutionellen Diskurses geblieben sind.

Die konstitutiven Charakteristiken des Diskurses wurden schrittweise gebildet. Ihr Auftreten wird in den frühen Stadien der Schulbildung aufgezeichnet. Die konstitutiven Charakteristiken des ukrainischen pädagogischen Diskurses sind der Bildungsbereich der Kommunikation, das mikrosoziale Kommunikationsumfeld, die Teilnehmer (Lehrer und Schüler / Studenten / Zuhörer), die Bedingungen (Bildungsräume, Bildungsgebiet) und die Organisation der Kommunikation. Die Sprache, die Werte und die Verhaltensregeln, die auf den Bestimmungen der christlichen Ethik gegründet sind, bilden die Ethnospezifität.

Der allgemeine Zweck des ukrainischen pädagogischen Diskurses während seiner Existenz bestand darin, um die jüngeren Mitglieder der Gesellschaft zu sozialisieren und die verantwortungsbewussten Bürger ihres Landes zu erziehen. Die spezifischen sozialgeschichtlichen Bedingungen haben diesen Zweck konkretisiert.

Die Kommunikationsmodelle im Ausbildungsbereich, die auf der Grundlage von ethnokommunikativen Traditionen entstanden haben, haben sich ständig unter dem Einfluss religiöser, politischer, sozioökonomischer und kultureller Faktoren verändert. 
Die Spezifität des ukrainischen pädagogischen Diskurses in verschiedenen Stadien der Entwicklung der Gesellschaft wurde durch verschiedene Faktoren beeinflusst: von der Antike bis zum 19. Jahrhundert hatte die Kirche den größten Einfluss, in dem Totalitarismus - die Politik und die Ideologie, in der industriellen und digitalen Epoche - die sozioökonomische Situation.

Die sprachstatusorientierte Interaktion im ukrainischen Ausbildungsbereich ist fast während der gesamten Geschichte durch die Hierarchie, die Strenge, die Direktivität und dem Zwang bezeichnend. Erst in den letzten Jahren sind die Trends der ukrainischen Ausbildung zu der Partnerschaft, die Personalisierung und die Gamification geworden. Der ukrainische pädagogische Diskurs zeigt die Dynamik des Volkes allgemein und die Dynamik der Ausbildung insbesondere.

\section{ZUSAMMENFASSUNG}

Die Entwicklung des ukrainischen pädagogischen Diskurses war kontinuierlich (ununterbrochen) und gleichzeitig ungleichmäßig. Er wurde maßgeblich von religiösen, politischen, sozioökonomischen und kulturellen Faktoren beeinflusst. Die konstitutiven Charakteristiken des Diskurses wurden schrittweise gebildet. Ihr Auftreten wird in den frühen Stadien der Schulbildung aufgezeichnet. Die konstitutiven Charakteristiken des ukrainischen pädagogischen Diskurses sind dem Bildungsbereich der Kommunikation, das mikrosoziale Kommunikationsumfeld, die Teilnehmer (Lehrer und Schüler / Studenten / Zuhörer), die Bedingungen (Bildungsräume, Bildungsgebiet) und die Organisation der Kommunikation. Die Sprache, die Werte und die Verhaltensregeln, die auf den Bestimmungen der christlichen Ethik gegründet sind, bilden die Ethnospezifität. Der Zweck des ukrainischen pädagogischen Diskurses während seiner Existenz bestand darin, um die jüngeren Mitglieder der Gesellschaft zu sozialisieren, aber er wurde durch sozialgeschichtliche Bedingungen konkretisiert.

\section{VERWEISE}

1. Карасик В.И. Языковой круг: личность, концепты, дискурс. Москва : Гнозис, 2004. 390 с.

2. Dijk T.A., van. Discourse and Context: A Sociocognitive Approach. New York : Cambridge University Press, 2008. 267 p.

3. Селіванова О.О. Лінгвістична енциклопедія. Полтава : Довкілля-К, 2010. 844 с.

4. Літопис руський / пер. Л.Є. Махновця. Київ : Дніпро, 1989. 591 с.

5. Крекотень В. Освітня реформа Петра Могили й утвердження бароко в українській поезії. Записки Наукового товариства імені Шевченка. Львів, 1992. Том CCXXIV. Праці Філологічної секції. C. 7-24. 
6. Микитась В. Давньоукраїнські студенти і професори. Київ : Абрис, 1994. 288 с.

7. Львівська братська школа. URL: https://uk.wikipedia.org/wiki/ Львівська_братська_школа.

8. Барт Р. Сад, Фурье, Лойола. Москва : Праксис, 2007. 256 с.

9. Попович М.В. Нарис історії культури України. Київ : АртЕк, $1998.727 \mathrm{c}$.

10. Шеретюк Р. «Благочестя і освіта»: просвітницькі ідеї Йосифа де Каласанса та їхній вплив на духовно-культурне життя Волині (XVII перша пол. XIX ст.). URL: http://nbuv.gov.ua/UJRN/apvtvi_2016_27_50.

11. Хропко П.П. Анатолій Свидницький. Анатолій Свидницький. Роман, оповідання, нариси / за ред. П. П. Хропка. Київ : Наукова думка, 1985. C. 5-26.

12. Сухомлинська О. Радянська педагогіка як ідеологія: спроба історичної реконструкції. URL: https://core.ac.uk/download/pdf/ 32308395.pdf.

13. Масенко Л. Мова радянського тоталітаризму. Київ : ТОВ «Видавництво "Кліо"», 2017. 240 с.

14. Сухомлинський В. Серце віддаю дітям. URL: https://mala.storinka.org/серце-віддаю-дітям-василь-сухомлинськийчитати-завантажити.html.

Information about the author: Kravets L. V., Dr (Hab) in Philology, Professor, Professor at the Department of Philology

Ferenc Rakoczi II Transcarpathian Hungarian College of Higher Education 6, Koshuta Square, Berehovo, Transcarpathian region, 90200, Ukraine 